\title{
Computational Disease Subtyping based on Joint Analysis of Clinical and Genomic Data
}

\author{
Diana Diaz ${ }^{1}$, Aliccia Bollig-Fischer ${ }^{2}$, Alexander Kotov ${ }^{1 *}$ \\ ${ }^{1}$ Department of Computer Science, Wayne State University, Detroit, USA \\ ${ }^{2}$ Department of Oncology, Wayne State University, School of Medicine and Karmanos Cancer Institute, Detroit, USA \\ * Correspondence: kotov@ wayne.edu, 5057 Woodward Ave., Suite 14001.6, Detroit, MI 48202, USA, (313) 577-9307
}

\begin{abstract}
Objective To investigate application of non-negative tensor decomposition for disease subtype discovery based on joint analysis of clinical and genomic data.

Data and Methods Somatic mutation profiles including 11,996 genes of 503 breast cancer patients from the Cancer Genome Atlas (TCGA) along with 11 clinical variables and markers of these patients were used to construct a binary third-order tensor. CANDECOMP/PARAFAC method was applied to decompose the constructed tensor into rank-one component tensors. Definitions of breast cancer verotypes were constructed from the patient, gene and clinical vectors corresponding to each component tensor. Patient membership proportions in the identified verotypes were utilized in a Cox proportional hazards model to predict their survival.

Results Qualitative evaluation of the verotypes obtained by tensor factorization indicates that they correspond to clinically meaningful breast cancer subtypes. While some components correspond to the known HER2- or ER-positive breast cancer subtypes, other components correspond to a variant of triple negative subtype and a cohort of patients with high mutation load of tumor suppressor genes. Quantitative evaluation indicates that the Cox model utilizing computationally discovered breast cancer verotypes is more accurate ( $\mathrm{AUC}=0.5796)$ at predicting patient survival than the Cox models utilizing random patient membership proportions in cancer subtypes $(\mathrm{AUC}=0.4056)$ as well as patient membership proportions in genotypes $(\mathrm{AUC}=0.4731)$ and phenotypes (AUC=0.5047) obtained by non-negative factorization of the somatic mutation and clinical matrices.

Conclusion Non-negative factorization of a binary tensor constructed from clinical and genomic data enables high-throughput discovery of breast cancer verotypes that are effective at predicting patient survival.

Keywords: Precision Medicine, Computational Disease Subtyping, Tensor Decomposition, Breast Cancer, Translational Bioinformatics
\end{abstract}

\section{BACKGROUND AND SIGNIFICANCE}

Successful transition into the era of precision medicine ${ }^{1,2}$ or screening, diagnostic, therapeutic and prognostic procedures that take into account individual variability of patients, requires comprehensive knowledge of complex relationships between molecular, biological and physiological processes in a human body. Stratification of patients into cohorts with a common biological basis and phenotypic manifestation of a particular disease is an important aspect of such knowledge. Remarkable advances in the next-generation sequencing technology coupled with widespread adoption of electronic health records (EHR) by healthcare providers in the United States have enabled collection of unprecedented amounts of genetic and clinical patient data, from which such knowledge can be discovered. Specifically, methods for high-throughput computational analysis of genetic and clinical data can help shed light on heterogeneous (molecular, biological and physiological) markers that are highly predictive of survival as well as the outcome of therapeutic agents and treatment strategies. Prior research along this direction has focused on the methods to analyze genetic and clinical data in isolation with the goal of identifying either phenotypes (i.e. sets of biomarkers that are more prevalent in individuals with a particular disease or condition than in the general population) ${ }^{3-9}$ or genotypes (i.e. DNA sequences that underlie specific diseases or traits $)^{10-14}$. In particular, the recently proposed computational methods for discovering EHR-based phenotypes have been successfully applied to patient cohort identification ${ }^{8}$ and determining the eligibility of patients for clinical trials. ${ }^{15}$ On the other hand, the mapping of the human genome has enabled computational genotyping methods, which typically combine clustering ${ }^{10,11,16}$ with data integration $^{12,17}$ and feature selection ${ }^{18,19}$ to identify the genes that are predictive of specific clinical outcomes. ${ }^{20-22}$ Previous research on personalized approaches to cancer treatment has primarily focused on genetic studies, including identification of pathogenic mutations of individual genes in cancer tumors, ${ }^{14}$ tumor stratification, ${ }^{22-25}$ functional diagnostics, ${ }^{26}$ and classification, ${ }^{27}$ as well as creating centralized resources, repositories and protocols for interpreting, validating, sharing and updating the results produced by these studies. ${ }^{28}$

Despite the immense progress in computational methods for analyzing clinical or genomic data, neither of these two types of methods alone can capture all aspects of the pathogenesis of complex diseases, ${ }^{29}$ such as cancer. The emergence of EHR-linked biobanks, such as those created by the Electronic Medical Records and Genomics (eMERGE) consortium, ${ }^{30}$ enabled computational methods to discover associations between specific diseases and genes ${ }^{31-34}$ through genome-wide association studies $(\text { GWAS })^{35}$ or between specific phenotypes and genes through phenomewide association studies (PheWAS). ${ }^{36,37}$

This paper reports the results of applying computational methods for high-throughput cancer subtyping based on the joint analysis of clinical and genomic data of breast cancer patients and utilizing the discovered subtypes for their survival prediction. Breast cancer is the most frequently diagnosed cancer and the second cause of cancer deaths in women. ${ }^{38}$ Due to a significant degree of variation, cancers are particularly difficult to treat, since patients with the same type of cancer and a similar set of symptoms can have different responses to the same treatment. ${ }^{39,40}$ One in three women with breast cancer detected by a mammogram unnecessarily undergo surgery or chemotherapy, ${ }^{41}$ which results in measurable toll of increased healthcare costs and unmeasurable emotional trauma. Therefore, methods for more comprehensive and accurate stratification of cancer patients would help oncology decisionmaking process, facilitate treatment selection and ultimately improve patient outcomes. There is a particular need for such methods in the case of women with early stages of breast cancer, since there is a great variety 


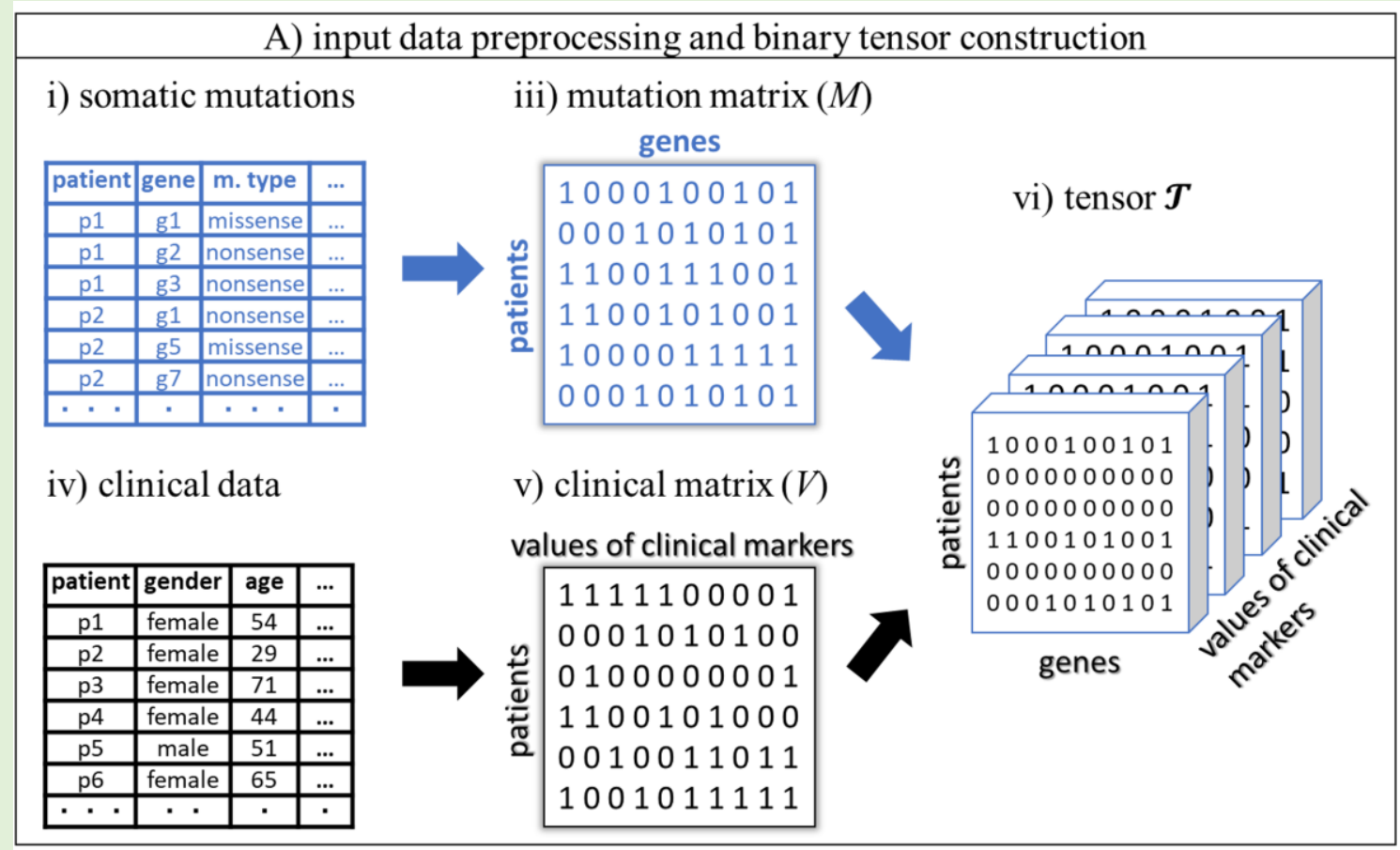

B) definitions of candidate cancer verotypes through $\mathrm{CP}$ decomposition of binary tensor

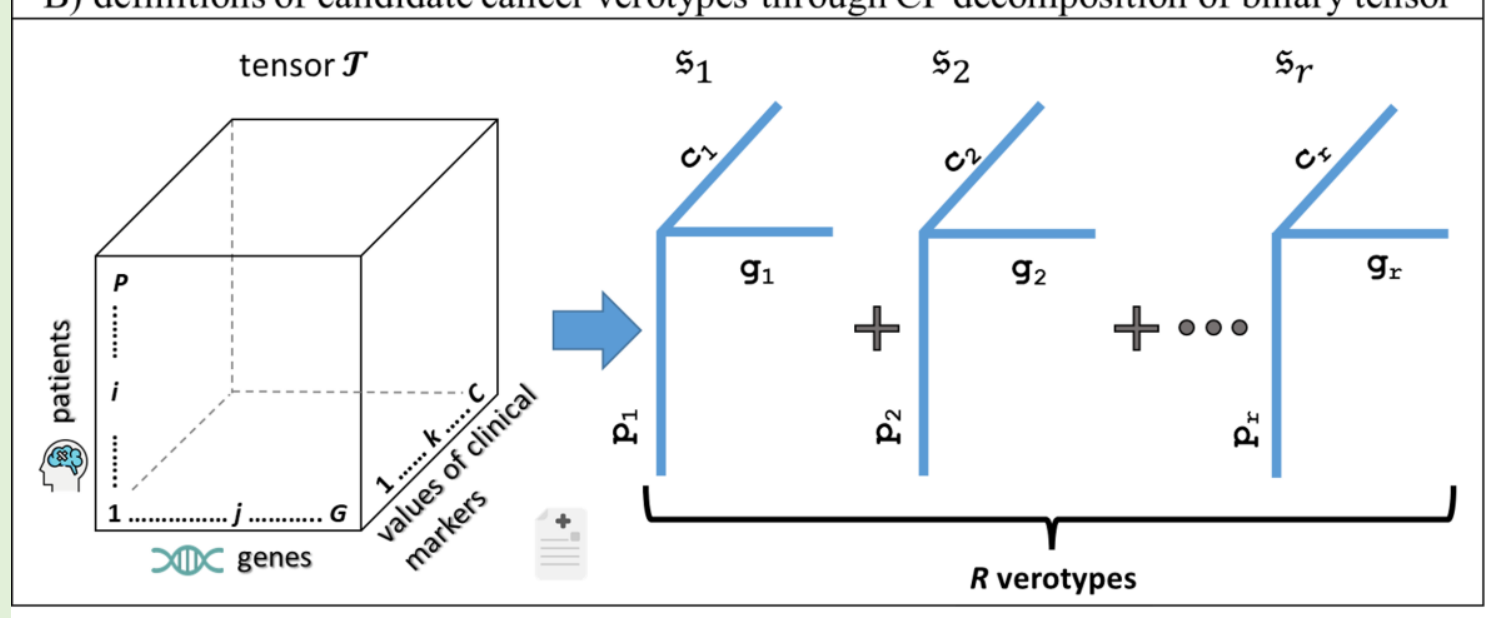

Figure 1. Two stages of the proposed CLIGEN pipeline: A) data preprocessing and construction of multi-modal threedimensional tensor $\mathcal{T}$. B) obtaining candidate verotypes via CP decomposition of tensor $\mathcal{T}$.

in treatments prescribed to those women, with some women being underand over-treated, as a result.

Since cancer development and progression are influenced by several factors, including germ-line or somatic tumor genetics, overall patient health as well as environmental or lifestyle factors, ${ }^{42}$ it is natural to assume that cancer subtypes should incorporate all these different modalities of patient data. However, existing integrative approaches are specific to one particular type of clinical data, such as chemistry evaluations, ${ }^{43}$ survival, ${ }^{20,44}$ or epidemiological data, ${ }^{45}$ and there has been relatively little research on computational methods for joint analysis of clinical and genomic data for disease subtyping.

To address this deficiency, we propose CLIGEN, a high-throughput pipeline for fully unsupervised disease subtyping based on CLInical and GENomic data. Specifically, we focus on the problem of identifying cohorts of patients, which share the same set of pathogenic gene mutations as well as the same values of clinical variables and markers. This problem is different from tumor stratification, which is based only on genetic information and is aimed at dividing the heterogeneous population of cancer tumors into biologically meaningful subtypes based on mRNA expression data ${ }^{22,24}$ or gene networks. ${ }^{23}$ Although genomescale molecular information provides an insight into biological processes driving tumor progression, cancer subtyping based on gene expression profiles alone has been shown to have limited correlation with clinical outcomes. ${ }^{46,47}$

In the first stage of the proposed pipeline, multi-modal patient data that includes somatic mutation profiles as well as clinical variables and markers is represented as a binary three-dimensional tensor. As differential measurements between a tumor and normal tissue, somatic 
Diaz et al., 24 Apr 2018 - preprint copy - BioRxiv

Table 1. List of notations used in this paper and their definitions

\begin{tabular}{|c|c|}
\hline \hline Symbol & Definition \\
\hline$M, V$ & somatic mutation and clinical matrices \\
$M_{i j}$ & cell of mutation matrix for patient $i$ and gene $j$ \\
$V_{i k}$ & cell of clinical matrix for patient $i$ and $k$-th value or interval of clinical markers \\
$\mathcal{T}$ & multi-modal binary CLIGEN tensor \\
$P, G, C$ & number of patients, genes as well as intervals and values of clinical markers \\
$i, j, k$ & indices of patients, genes, and clinical markers. \\
$t_{i j k}$ & value of tensor cell for the $i$-th patient, $j$-th gene, and $k$-th value or interval of clinical \\
$\circ$ & markers \\
$R$ & outer product of two vectors \\
$\mathfrak{s}_{r}$ & number of tensor components \\
$p_{r}, g_{r}, c_{r}$ & patient, gene and clinical factor vectors \\
\hline \hline
\end{tabular}

mutation profiles are more suitable for disease subtyping than other types of 'omics' data, which are absolute measurements for each patient. Furthermore, somatic mutations capture causal genetic events underlying tumor progression, whereas mRNA or protein expression profiles are functional readouts of the current cell state and can be influenced by external factors that are unrelated to tumor biology.

In the second stage of the proposed pipeline, non-negative tensor decomposition is applied to identify latent factors in each modality of the constructed tensor. These latent factors correspond to the frequently cooccurring combinations of gene mutations and clinical markers in patients with a particular complex disease, such as cancer. We hypothesize that the proposed pipeline enables discovery of cancer verotypes (clinical disease subtypes combining phenotypes and genotypes). ${ }^{48}$ To validate this hypothesis, we applied the proposed framework to discover breast cancer verotypes based on the clinical and genetic data in the Cancer Genome Atlas (TCGA) ${ }^{49}$ and experimentally demonstrate that the discovered breast cancer verotypes can provide actionable insights, such as patient survival prognosis, to clinicians at the point of care.

\section{DATA AND METHODS Dataset description}

We used somatic mutation (non-silent mutation from the whole exome sequencing level 3) profiles and clinical data of breast cancer patients from The Cancer Genome Atlas (TCGA) ${ }^{49}$ for all experiments in this work (data downloaded from cBioportal on 9 April 2017). ${ }^{50} \mathrm{We}$ considered only the patients for whom both somatic mutation and clinical data were available and discarded the patients with fewer than 10 somatic mutations. This resulted in a dataset combining information about mutations in 11,996 genes and 70 dichotomized values of 11 clinical variables and markers for 503 patients.

\section{Somatic mutations}

The somatic mutation table consists of 37 columns and 34032 registries. A registry in this table indicates a mutation in the gene reported in the column 'Hugo Symbol' for the sample in the column 'Tumor Sample Barcode'. Additional details on processing and organization of these data are available in. ${ }^{50}$ In this work, we constructed patient mutation profiles as binary vectors, in which a bit is set, if the patient's gene corresponding to that position in the vector harbors a mutation.

\section{Clinical variables and markers}

The clinical variables and markers (without loss of generality, further referred to as clinical markers) in TCGA include the age of diagnosis with cancer, gender, estrogen receptor (ER) status, progesterone receptor (PR) status, human epidermal growth factor receptor 2 (HER2) final status, American Joint Committee on Cancer (AJCC) breast cancer stage, AJCC coded tumor stage (T), AJCC coded lymph node stage (N), AJCC coded metastasis stage (M), IHC (immunohistochemistry) expression level and PAM50 profile.

\section{Proposed pipeline}

CLIGEN, the proposed pipeline for unsupervised verotyping of complex diseases, is illustrated in Figure 1. The input to the pipeline consists of genetic and clinical data of the patients with the same complex disease (e.g. cancer). The output of the pipeline is a set of definitions of disease subtypes characterized by genetic and clinical markers. The pipeline consists of the two stages: 1) data preprocessing and tensor construction and 2) non-negative decomposition of the constructed tensor to obtain cancer subtypes. Detailed descriptions of each stage are provided below and the notations used in these descriptions are summarized in Table 1. Code for CLIGEN is available at http://www.cs.wayne.edu/dmd/cligen/.

\section{Data preprocessing}

The first stage of the proposed pipeline, illustrated in Figure 1A, involves preprocessing the input genomic and clinical patient data, to create a combined representation for subsequent analysis.

\section{Somatic mutation data}

Somatic mutation datasets typically take the form of mutation tables, in which the rows correspond to mutations and the columns describe the type of each mutation and its location (Figure 1Ai). Based on the input mutation table, CLIGEN constructs a binary mutation matrix $M$ with patients as rows and genes as columns. The value of 1 in the cell $M_{i j}$ of matrix $M$ indicates that patient $i$ has at least one non-silent mutation (i.e., a mutation of any of the following types: missense mutation, nonsense mutation, non-stop mutation, in-frame insertion, in-frame deletion, or frameshift mutation) in gene $j$, while the value of 0 indicates that patient $i$ has no mutations in gene $j$.

\section{Clinical data}

Continuous clinical variables or markers, such as the age of diagnosis, were discretized into intervals with a total of $n$ combined intervals of all continuous markers and levels of all discrete markers in the entire dataset. The values of clinical markers for each patient were represented as a 
Diaz et al., 24 Apr 2018 - preprint copy - BioRxiv

binary clinical matrix $V$ with patients as rows and intervals of continuous or levels of discrete clinical markers as columns. The value of 1 in the cell $V_{i k}$ of matrix $V$ indicates that patient $i$ has a $k$ th level or interval of a discrete or continuous clinical marker.

\section{Tensor construction}

Clinical and mutation matrices are combined to create a threedimensional binary CLIGEN tensor (i.e. multidimensional array) $\mathcal{T}$ representing interactions between clinical markers and genetic mutations. The first dimension (i.e. mode) of tensor $\mathcal{T}$ corresponds to patient data, while the other two dimensions correspond to the values of clinical markers and genes (Figure 1B). Each element $t_{i j k}$ of the tensor $\mathcal{T}$ has the value of 1 , if the $i$ th patient has at least one mutation in gene $j$ and $k$ th value of clinical markers and 0 otherwise.

Slicing tensor $\mathcal{T}$ along each of its three modes yields the following views:

1. Patient mode: each slice is a matrix of co-occurrences of mutations and clinical markers for a particular patient. For instance, if a patient's health records indicate stage I breast cancer and her mutation profile indicates a mutation in gene TP53, then a cell at the row for the "Stage I" clinical variable and the column for the TP53 gene in the matrix corresponding to the tensor slice for this patient will have a value of 1. The cells in the same column and the rows for "Stage II", "Stage III" and "Stage IV" will have the value of 0 .

2. Gene mode: each slice is a matrix with patients as rows and clinical markers as columns, which shows how a mutation in a particular gene is correlated with clinical markers in different patients. Such matrix can be considered as a summary of phenotypic manifestations of a particular gene mutation.

3. Clinical mode: each slice is a matrix with patients as rows and genes as columns, which shows how gene mutations in different patients are correlated with a particular clinical marker. Such matrix can be considered as a summary of genetic markers for a simple phenotype.

\section{Obtaining candidate verotype definitions through tensor factorization}

Tensor decomposition ${ }^{51}$ is a powerful mathematical technique that has been successfully applied in different domains ranging from psychology and neuroscience to computer vision. ${ }^{52}$ In biomedical informatics, tensor decomposition has proven to be useful for understanding cellular states ${ }^{53}$ and biological processes, ${ }^{54}$ in addition to EHR-based phenotyping ${ }^{3-5}$. Tensor decomposition has several advantages over matrix factorization. First, tensors explicitly account for the multiway structure of the data that is otherwise lost, when a tensor is converted into a matrix by collapsing some of its modes. Second, some tensor decomposition methods guarantee uniqueness of the optimal solution even for very sparse tensors. The two most widely used tensor decomposition methods are the Tucker method $^{55}$ and $\mathrm{CP}$, which stands for Canonical Decomposition (CANDECOMP), ${ }^{56}$ also known as Parallel Factor Analysis (PARAFAC) ${ }^{57} \mathrm{CP}$ decomposes a tensor into a linear combination of rank-one tensor components. ${ }^{56}$ Each component of the CLIGEN binary tensor consists of vectors corresponding to each mode, ${ }^{51}$ with each vector corresponding to groups of frequently co-occurring genes, clinical markers or patients. The $\mathrm{CP}$ decomposition can be considered as a special case of the Tucker decomposition when the size of each modality of the core array is the same and the only non-zero elements in the core are the elements along the main diagonal. ${ }^{51}$ An important property of $\mathrm{CP}$ decomposition is that the restriction imposed on the Tucker core leads to the uniqueness of the optimal solution. ${ }^{51}$

Specifically, CP decomposition of a third-order CLIGEN tensor $\mathcal{T} \in$ $\mathbb{R}^{G \times P \times C}$ with dimensions corresponding to genes, patients, and clinical markers is formally defined as:

$$
\mathcal{T} \approx \hat{\mathcal{T}}=\llbracket \lambda ; \mathbf{P}, \mathbf{G}, \mathbf{C} \rrbracket=\sum_{r=1}^{R} \lambda_{r} \cdot \mathfrak{S}_{r}=\sum_{r=1}^{R} \lambda_{r} \cdot \mathbf{p}_{r} \circ \mathbf{g}_{r} \circ \mathbf{c}_{r}
$$

where $R$ is the number of component rank-one tensors $\mathfrak{s}_{r}$ that tensor $\mathcal{T}$ is decomposed into and $\lambda_{r} \in \mathbb{R}$ is the weight of the $r$ th component. Each component is associated with three length normalized non-negative vectors: a patient vector $\mathbf{p}_{r} \in \mathbb{R}^{P}$, a gene vector $\mathbf{g}_{r} \in \mathbb{R}^{G}$ and a clinical vector $\mathbf{c}_{r} \in \mathbb{R}^{C}$. Patient, gene and clinical vectors for all tensor components correspond to the columns of the patient, gene and clinical factor matrices $\mathbf{P}, \mathbf{G}, \mathbf{C}$.

$\mathrm{CP}$ decomposition is obtained by solving the following optimization problem:

$$
\min _{\hat{\mathcal{T}}}\|\mathcal{T}-\hat{\mathcal{T}}\|_{F}
$$

aimed at finding the best approximation $\hat{\mathcal{T}}$ of each element $t_{i j k}$ of the original tensor $\mathcal{T}$ from the factor vectors corresponding to the components tensors as follows:

$t_{i j k} \approx \sum_{r=1}^{R} \lambda_{r} p_{i r} g_{j r} c_{k r}$ for $i=1, \ldots, P, j=1, \ldots, G$, and $k=1, \ldots, C$

Verotype definitions are constructed from patient, gene and clinical factor vectors corresponding to each of the component tensors obtained by $\mathrm{CP}$ decomposition of the CLIGEN tensor. Each element in the vectors corresponding to gene and clinical tensor modes can be interpreted as an importance of a gene or clinical marker to a verotype. The prevalence of a verotype among patients is calculated as a proportion of non-zero entries in the patient vector corresponding to a given verotype.

\section{RESULTS}

We performed both qualitative and quantitative evaluation of breast cancer subtypes derived from TCGA breast cancer dataset ${ }^{50}$ using the proposed pipeline.

\section{Quantitative evaluation}

Quantitative evaluation was conducted for the task of patient survival prognosis, which is important for personalizing cancer treatment. ${ }^{58}$ Specifically, we compared Cox proportional hazards models that use the following predictors for survival prognosis of breast cancer patients:

M1: membership proportions of a patient in breast cancer verotypes, which correspond to a row in the patient factor matrix $\mathbf{P}$ obtained by the proposed pipeline;

M2: membership proportions of a patient in breast cancer genotypes, which correspond to a row in matrix $\mathbf{P}$ obtained by nonnegative factorization of the somatic mutation matrix $M$ (as in [23] but without gene network smoothing), as shown in Figure 2;

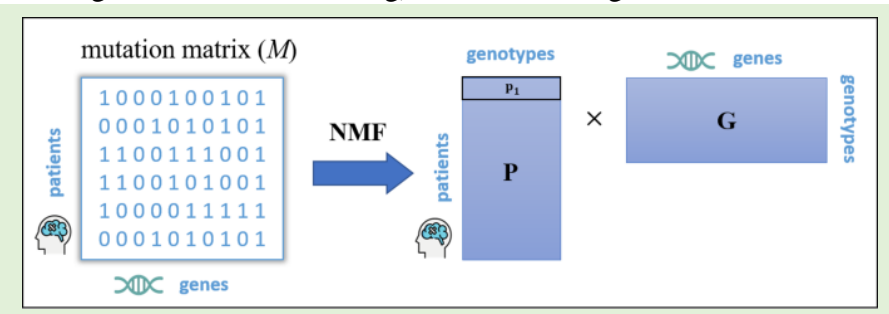

Figure 2. Obtaining genotypes through non-negative factorization of a binary somatic mutation matrix. The rows in matrix $P$ correspond to a vector of genotype memberships (e.g. $p_{1}$ is a vector of genotype memberships for the first patient). Rows in matrix $\mathbf{G}$ correspond to genotype definitions. 
M3: membership proportions of a patient in breast cancer phenotypes, which correspond to a row in the patient factor matrix obtained by non-negative factorization of the clinical matrix $V$;

M4: random membership proportions of a patient in each number of breast cancer subtypes

In the first experiment, we compared the accuracy of the Cox models using each of the above predictors for survival prognosis of breast cancer patients, while in the second experiment, we compared the goodness of fit of these models.

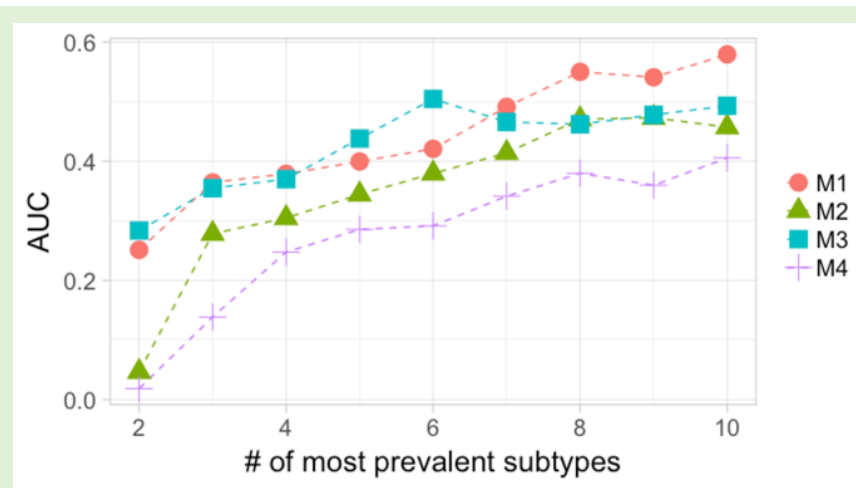

Figure 3. AUC of Cox models for breast cancer patient survival prediction that utilize patient membership proportions in most prevalent verotypes obtained by the proposed pipeline (M1), patient membership proportions in genotypes (M2) obtained by NMF of binary somatic mutation matrix, phenotypes (M3) obtained by NMF of binary clinical matrix as predictors, and random patient membership proportions (M4).

\section{Accuracy of survival prognosis}

In the first experiment, we compared the area under the ROC curve (AUC) for the models M1-M4. We used 10 random splits, such that each split divides the TCGA patient data into two halves (50\% for testing and $50 \%$ for training), as experimental design. The Cox models were estimated using the data in the training splits and evaluated using the data in the testing splits.

The plot of AUC values for models M1-M3 micro-averaged over splits by varying the number of the most prevalent cancer verotypes, genotypes and phenotypes is shown in Figure 3, from which two major conclusions can be drawn. First, the Cox regression model that utilizes patient membership proportions in verotypes obtained by CLIGEN (M1) is consistently more accurate at predicting breast cancer survival than the Cox model that uses membership proportions in genotypes obtained by NMF (M2) and phenotypes obtained by NMF (M3), which indicates the importance of taking into account both clinical and genomic data when determining cancer subtypes. In particular, the Cox model utilizing patient verotype membership proportions as predictors achieved the highest AUC of 0.5796, when 10 most prevalent verotypes were used, while the Cox model utilizing patient genotype memberships as predictors achieved the highest AUC of 0.4731 , when the 9 most prevalent genotypes were used, and the Cox model utilizing patient phenotype memberships as predictors achieved the highest AUC of 0.5047, when the 6 most prevalent phenotypes were used.

Second, the Cox models utilizing patient membership proportions in the top- $k$ most prevalent verotypes derived by CLIGEN as well as the phenotypes and genotypes derived by NMF are all more accurate at predicting breast cancer survival than the baseline Cox model utilizing random patient membership proportions in the same number of cancer subtypes (AUC $=0.4056$ ).

\section{Goodness of fit}

In the second experiment, we compared the goodness of fit of the models M1-M4 estimated on the entire TCGA dataset. The p-values of Log-rank and Wald tests of these models are summarized in Table 2. Both tests indicate that patient membership proportions in verotypes derived by CLIGEN are more statistically significant predictors of breast cancer patient survival than membership proportions in breast cancer phenotypes, which in turn are more statistically significant predictors than random patient membership proportions and membership proportions in genotypes derived by NMF.

Table 2. p-values of Log-rank and Wald tests of the Cox proportional hazard models utilizing patient membership proportions in verotypes (M1), genotypes (M2), phenotypes (M3), and random membership assignment (M4) as predictors.

\begin{tabular}{|c|c|c|}
\hline \hline Model & Log-rank test & Wald test \\
\hline M1 & $8.327 \mathrm{e}-15$ & 0.000082 \\
M2 & 0.315 & 0.3772 \\
M3 & 0.0007 & 0.0124 \\
M4 & $1.29 \mathrm{e}-5$ & 0.00012 \\
\hline
\end{tabular}

Kaplan-Meier survival plots for the 4 most prevalent breast cancer subtypes obtained using CLIGEN and NMF are shown in Figure 4. As follows from Figure 4, the most prevalent subtypes obtained using the proposed pipeline are more distinct in terms of patient survival dynamics ( $p=0.0493)$ than the most prevalent subtypes obtained using genotypes $(p=0.241)$ and phenotypes $(p=0.2073)$.

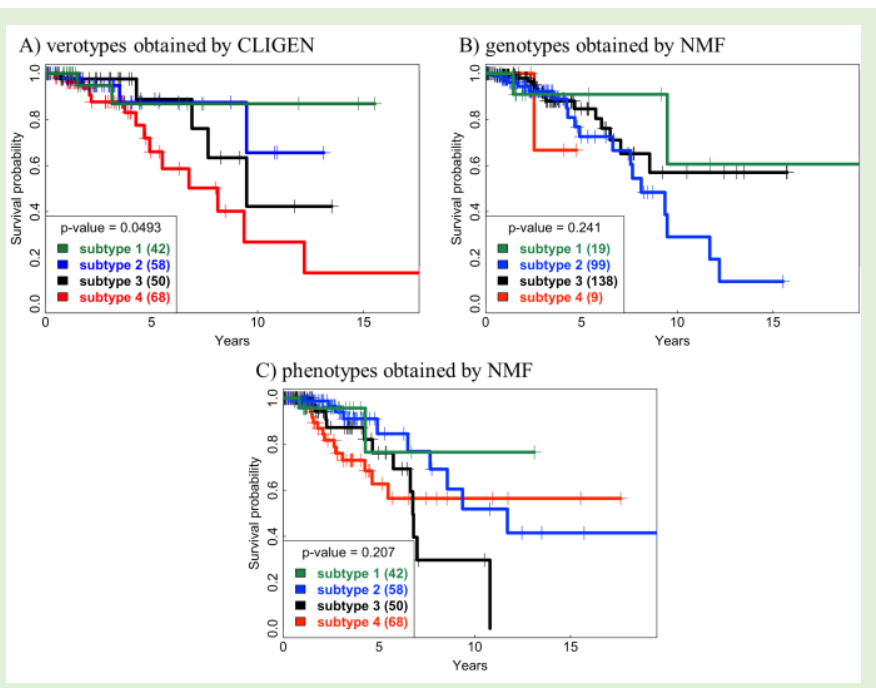

Figure 4. Kaplan-Meier survival plots for the 4 most prevalent: A) verotypes obtained using CLIGEN, B) genotypes obtained using NMF, C) phenotypes obtained using NMF.

\section{Qualitative evaluation}

An oncologist performed qualitative evaluation of breast cancer verotypes obtained by CP decomposition of the binary CLIGEN tensor constructed from the TCGA dataset into 10 components, since this decomposition results in the most accurate prediction of cancer survival. An enrichment analysis of the list of genes associated with each verotype was performed using the Ingenuity Systems Upstream Analysis tool. ${ }^{59}$ 
Diaz et al., 24 Apr 2018 - preprint copy - BioRxiv

A) verotype characterized by a high mutation load, which may be associated with a mutation in a DNA repair gene pathway(s).

\begin{tabular}{|l|l|l|l|}
\hline \multicolumn{4}{|l|}{ Component 1 } \\
\hline \hline \multirow{2}{*}{$2.761 \%$ of patients } & \multicolumn{3}{|l|}{ Top genes } \\
\cline { 3 - 5 } & TP53 & DNAH7 & MACF1 \\
\cline { 2 - 5 } & PIK3CA & MUC17 & CROCCP2 \\
\cline { 2 - 5 } & TTN & SSPO & ASXL3 \\
\hline \multirow{2}{*}{$0 \%$ of clinical variables } & BRCA1 & FAT3 & SYNE1 \\
\cline { 3 - 5 } & USH2A & OBSCN & DST \\
\cline { 2 - 5 } & Unknown & FLG & RYR2 \\
\cline { 2 - 5 } & MUC16 & KMT2C & LRP2 \\
\hline
\end{tabular}

C) verotype characterized by overexpression of progesterone receptor $(\mathrm{PR})$ and estrogen receptor alpha-positive $(\mathrm{ER}+)$. It typically has the worst survival prognosis relative to other breast cancer subtypes.

\begin{tabular}{|l|l|l|l|}
\hline \multicolumn{4}{|c|}{ Component 6 } \\
\hline \hline \multirow{2nnn}{*}{$35.503 \%$ of patients } & \multicolumn{3}{|c|}{ Top genes } \\
\cline { 4 - 5 } & TP53 & SMG8 & SAMD9 \\
\hline AJCC.StageStage.III & KDM5C & MTO1 & ITGA1 \\
\hline AJCC.StageStage.IIIB & NCOA6 & KL & WDR52 \\
\cline { 4 - 5 } PR.StatusPositive & HYDIN & ZFHX3 & FXR1 \\
\hline PPHLN1 & SYNE3 & SCN7A \\
\hline ER.StatusPositive & BTD & ATG16L1 & BID \\
\hline
\end{tabular}

Figure 5. Examples of verotypes identified by the proposed pipeline.
B) triple negative breast cancer verotype defined by the lack of estrogen receptor (ER), progesterone receptor (PR) and the human epidermal growth factor receptor (HER2) expression

\begin{tabular}{|c|c|c|c|}
\hline \multicolumn{4}{|c|}{ Component 5} \\
\hline \multirow{2}{*}{$36.489 \%$ of patients } & \multicolumn{3}{|c|}{ Top genes } \\
\hline & TTN & UBR4 & DNAH11 \\
\hline Metastasis.M1 & MUC16 & HDAC6 & UBA1 \\
\hline & CROCCP2 & SPI1 & ELL3 \\
\hline AJcC.Stagestage.X & KMT2C & SETD2 & OR2B2 \\
\hline Tumor.TX & WDFY3 & GON4L & UBA6 \\
\hline ER.StatusNegative & SYNE2 & ZNF821 & SPEN \\
\hline & VCAN & LRP2 & BLM \\
\hline
\end{tabular}

D) verotype characterized by overexpression of the human epidermal growth factor receptor (HER2) oncogene and responsive to HER2targeted inhibitors

\begin{tabular}{|c|c|c|c|}
\hline \multicolumn{4}{|c|}{ Component 9} \\
\hline \multirow{2}{*}{$48.521 \%$ of patients } & \multicolumn{3}{|c|}{ Top genes } \\
\hline & TTN & LRP2 & EMR2 \\
\hline ER.StatusPositive & USH2A & ATP1A4 & RP11-32B5.1 \\
\hline HER2.StatusPositive & MUC16 & TLN2 & ZFHX3 \\
\hline Metastasis.CodedNegative & RYR2 & ERBB3 & TRIP11 \\
\hline Nodes.DetailsPositive & FLG & COL6A3 & SRCAP \\
\hline TumorT3 & WDFY3 & DNAH11 & MEP1A \\
\hline & GPR98 & DNAH8 & PEG3 \\
\hline
\end{tabular}

\section{Examination of verotypes}

Each breast cancer verotype obtained by CLIGEN was analyzed and compared with the known breast cancer subtypes. Component 1 (shown in Figure 5A) corresponds to a small cohort of patients with a high mutation load. Further investigation of this verotype revealed a large number of mutations in the tumor suppressor genes (BRCA1, BRCA2, TP53, PTEN, RB1) that participate in DNA repair, which indicates that the high mutation load may be associated with a mutation in a DNA repair gene pathway(s). As follows from Figure 6, for each sample, these mutations were mutually exclusive. Further investigation of these genes can elucidate biological process(es) underlying these mutations.

Component 5 (shown in Figure 5B) appears to correspond to a subtype of the triple negative breast cancer (TNBC), which is a defined by the lack of ER, PR and HER2 expression. Molecular aberrations driving this breast cancer subtype remain undefined and patients with this subtype of breast cancer have the worst prognosis relative to the patients with any other known breast cancer subtype. Component 6 (shown in Figure 5C) corresponds to the subtype of progesterone receptor and estrogen receptor alpha-positive $(\mathrm{ER}+)$ cancers, that are responsive to anti-ER therapies. Based on the associated clinical markers, Component 9 (shown in Figure 5D) appears to be related to the known subtype of breast cancer that is driven by overexpression of the epidermal growth factor receptor oncogene (HER2) and responsive to HER2-targeted inhibitors.

Triple negative Breast Cancer

Further analysis of putative cancer driver genes, potentially activated or inactivated by mutations associated with the TNBC-related fifth component resulted in significant enrichment of genes with a role in signaling networks that promote the function of cancer stem-like cells (CSCs), i.e., downstream of transcription factor TWIST1, and alternative
mRNA splicing, i.e., downstream of serine and arginine-rich splicing factor SRSF2. CSCs are identified in patient TNBC tumors as a fraction of self-renewing, tumor-initiating cancer cells that also give rise to drug resistance and metastatic recurrence. ${ }^{60-62}$ Alternative mRNA splicing has also been implicated in maintaining and generating CSCs. ${ }^{63}$

\section{DISCUSSION}

Methods for integrative analysis of genomic and clinical data face a common challenge of dealing with large volumes of data. By utilizing sparse representations and inexpensive linear algebra operations, tensor factorization methods effectively address this challenge. Successful application of tensor decomposition in different domains led to further research into efficient optimization methods for tensor decomposition, ${ }^{64}$ which makes tensor decomposition a method of choice for highthroughput disease subtyping.

Since tensor factorization models are parametric, selecting the optimal number of components for $\mathrm{CP}$ decomposition of the binary tensor (i.e. model order estimation) is an important practical aspect of the proposed pipeline. Too few components typically result in general verotype definitions, which may combine several actual disease subtypes. Too many components typically result in specific verotype definitions, which may split the actual disease subtypes. It is important to point out that, in terms of the number of model parameters, $\mathrm{CP}$ decomposition, which assumes that the number of components is the same per each tensor mode, has an advantage over Tucker decomposition, which requires specifying the number of components per each mode. While it is known that the number of components that minimizes the reconstruction error of the original tensor from its components is equal to the rank of a given tensor, ${ }^{51,65}$ finding tensor rank is an NP-complete problem. ${ }^{66}$ Even if the rank of a tensor is known, the number of components that minimizes 
Diaz et al., 24 Apr 2018 - preprint copy - BioRxiv

reconstruction error may not result in the best accuracy for a particular task, such as survival prediction. Therefore, the optimal number of components is typically determined using heuristics, such as core consistency diagnostic, ${ }^{67}$ cross validation ${ }^{68}$ (as was done in this work) or hierarchical Bayesian approach, ${ }^{69}$ if a suitable prior can be defined.

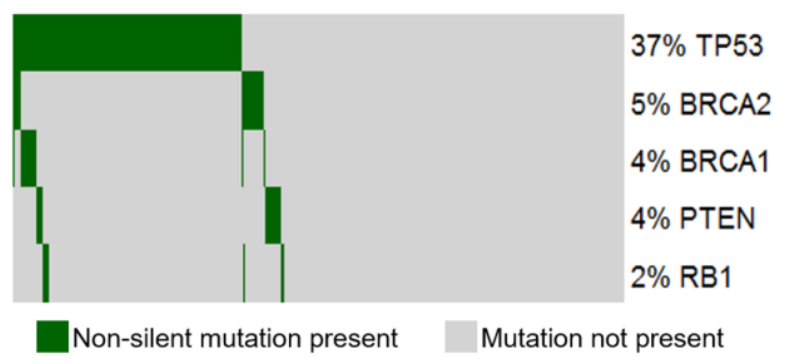

Figure 6. Mutual exclusivity across the characteristic genes of one of the verotypes identified by the proposed pipeline.

Tensor construction is another aspect of the proposed approach with possible variations. In this work, we used the presence or absence of nonsilent gene mutation as a single genetic signature of patients. However, it is possible to use other types of genomic data, such as gene expression or copy number variation. It is also possible to construct a count tensor, instead of a binary tensor, by taking into account both the type of mutations and the number of mutations per gene, which we leave for future work.

\section{CONCLUSION}

In this paper, we introduced CLIGEN, a novel machine learning based pipeline for unsupervised disease subtype discovery based on nonnegative decomposition of a binary tensor combining clinical and somatic mutation patient data, and applied the proposed pipeline to breast cancer subtyping. Qualitative and quantitative evaluation of the discovered breast cancer subtypes indicates that representation of clinical and genomic patient data as a binary tensor and its subsequent nonnegative decomposition is an effective computational approach to highthroughput disease subtyping for precision medicine. In particular, our proposed pipeline was not only able to identify known breast cancer subtypes (HER2+ and ER+), but also elucidated new possible characteristics of a complex breast cancer subtype (triple negative), which provides an opportunity for further research to define new cancer subtypes. We also demonstrated that patient membership proportions in the discovered breast cancer verotypes are more effective predictors of breast cancer survival than patient membership proportions in computationally identified genotypes and phenotypes.

Although the proposed pipeline was evaluated using the data of breast cancer patients, it can, in principle, be applied to computational subtyping of other cancers and complex diseases.

\section{Acknowledgments}

Results presented in this paper are based on the data in The Cancer Genome Atlas managed by the NCI and NHGRI. We would like to thank Dr. Sorin Drăghici for the helpful suggestions regarding this manuscript.

\section{REFERENCES}

1 Ashley EA. The Precision Medicine Initiative. JAMA 2015;313:2119. doi:10.1001/jama.2015.3595

2 Collins FS, Varmus H. A New Initiative on Precision Medicine. N Engl J Med 2015;372:793-5. doi:10.1056/NEJMp1500523
3 Ho JC, Ghosh J, Steinhubl SR, et al. Limestone: High-throughput candidate phenotype generation via tensor factorization. J Biomed Inform 2014;52:199-211. doi:10.1016/j.jbi.2014.07.001

4 Ho JC, Ghosh J, Sun J. Marble: high-throughput phenotyping from electronic health records via sparse nonnegative tensor factorization. ACM 2014. 115-24. doi:10.1145/2623330.2623658

5 Wang Y, Chen R, Ghosh J, et al. Rubik: Knowledge Guided Tensor Factorization and Completion for Health Data Analytics. ACM 2015. 1265-74. doi:10.1145/2783258.2783395

6 Hripcsak G, Albers DJ. Next-generation phenotyping of electronic health records. J Am Med Informatics Assoc 2013;20:117-21. doi:10.1136/amiajnl-2012-001145

7 Pathak J, Kho AN, Denny JC. Electronic health records-driven phenotyping: challenges, recent advances, and perspectives. J Am Med Informatics Assoc 2017;:e206-11. doi:10.1136/amiajnl-2013002428

8 Shivade C, Raghavan P, Fosler-Lussier E, et al. A review of approaches to identifying patient phenotype cohorts using electronic health records. J Am Med Inform Assoc 2014;21:221-30. doi:10.1136/amiajnl-2013-001935

9 Chen Y, Carroll RJ, Hinz ERM, et al. Applying active learning to high-throughput phenotyping algorithms for electronic health records data. J Am Med Informatics Assoc 2013;20:e253--e259. doi:10.1136/amiajnl-2013-001945

10 Eisen MB, Spellman PT, Brown PO, et al. Cluster analysis and display of genome-wide expression patterns. Proc Natl Acad Sci 1998;95:14863-8.

11 Kim E-Y, Kim S-Y, Ashlock D, et al. MULTI-K: accurate classification of microarray subtypes using ensemble k-means clustering. BMC Bioinformatics 2009;10:260. doi:10.1186/14712105-10-260

12 Wang B, Mezlini AM, Demir F, et al. Similarity network fusion for aggregating data types on a genomic scale. Nat Methods 2014; advance on. doi:10.1038/nmeth.2810

13 Hsu JJ, Finkelstein DM, Schoenfeld DA. Outcome-Driven Cluster Analysis with Application to Microarray Data. PLoS One 2015;10:e0141874. doi:10.1371/journal.pone.0141874

14 Lawrence MS, Stojanov P, Mermel $\mathrm{CH}$, et al. Discovery and saturation analysis of cancer genes across 21 tumour types. Nature 2014;505:495-501. doi:10.1038/nature12912

15 Richesson RL, Hammond WE, Nahm M, et al. Electronic health records based phenotyping in next-generation clinical trials: a perspective from the NIH Health Care Systems Collaboratory. J Am Med Inform Assoc 2013;20:e226-31. doi:10.1136/amiajnl-2013001926

16 Sinha A, Shen Z, Song Y, et al. An Overview of Microsoft Academic Service (MAS) and Applications. Proc 24th Int Conf World Wide Web - WWW '15 Companion 2015;:243-6. doi:10.1145/2740908.2742839

17 Nguyen T, Tagett R, Diaz D, et al. A novel approach for data integration and disease subtyping. Genome Res 2017;27:2025-39. doi:10.1101/gr.215129.116

18 Shai R, Shi T, Kremen TJ, et al. Gene expression profiling identifies molecular subtypes of gliomas. Oncogene 2003;22:4918-23. doi:10.1038/sj.onc. 1206753

19 Diaz D, Donato M, Nguyen T, et al. MicroRNA-augmented pathways(mirAP) and their applications to pathway analysis and 
Diaz et al., 24 Apr 2018 - preprint copy - BioRxiv

disease subtyping. Pac Symp Biocomput 2016;22.

20 Bair E, Tibshirani R. Semi-Supervised Methods to Predict Patient Survival from Gene Expression Data. PLOS Biol 2004;2:e108. doi:10.1371/journal.pbio.0020108

21 Collisson EA, Sadanandam A, Olson P, et al. Subtypes of pancreatic ductal adenocarcinoma and their differing responses to therapy. Nat Med 2011;17:500-3. doi:10.1038/nm.2344

22 Reis-Filho JS, Pusztai L. Gene expression profiling in breast cancer: classification, prognostication, and prediction. Lancet 2011;378:1812-23. doi:10.1016/S0140-6736(11)61539-0

23 Hofree M, Shen JP, Carter H, et al. Network-based stratification of tumor mutations. Nat Methods 2013;10:1108-15. doi:10.1038/nmeth.2651

24 Esteva FJ, Sahin AA, Cristofanilli M, et al. Prognostic role of a multigene reverse transcriptase-PCR assay in patients with nodenegative breast cancer not receiving adjuvant systemic therapy. Clin Cancer Res 2005;11:3315-9. doi:10.1158/1078-0432.CCR-041707

25 Reis-Filho JS, Pusztai L. Gene expression profiling in breast cancer: classification, prognostication, and prediction. Lancet 2011;378:1812-23. doi:10.1016/S0140-6736(11)61539-0

26 Greenman C, Stephens P, Smith R, et al. Patterns of somatic mutation in human cancer genomes. Nature 2007;446:153-8. doi:10.1038/nature05610

27 Friedman AA, Letai A, Fisher DE, et al. Precision medicine for cancer with next-generation functional diagnostics. Nat Rev Cancer 2015; 15:747-56. doi:10.1038/nrc4015

28 Roychowdhury S, Chinnaiyan AM. Advancing precision medicine for prostate cancer through genomics. J Clin Oncol 2013;31:186673. doi:10.1200/JCO.2012.45.3662

29 Rehm HL, Berg JS, Brooks LD, et al. ClinGen - The Clinical Genome Resource. N Engl J Med 2015;372:2235-42. doi:10.1056/NEJMsr1406261

30 Robinson PN. Deep phenotyping for precision medicine. Hum. Mutat. 2012;33:777-80. doi:10.1002/humu.22080

31 McCarty CA, Chisholm RL, Chute CG, et al. The eMERGE Network: A consortium of biorepositories linked to electronic medical records data for conducting genomic studies. BMC Med Genomics 2011;4:13. doi:10.1186/1755-8794-4-13

32 Botstein D, Risch N. Discovering genotypes underlying human phenotypes: past successes for mendelian disease, future approaches for complex disease. Nat Genet 2003;33 Suppl:228-37. doi:10.1038/ng1090

33 Kullo IJ, Fan J, Pathak J, et al. Leveraging informatics for genetic studies: use of the electronic medical record to enable a genomewide association study of peripheral arterial disease. J Am Med Informatics Assoc 2010;17:568-74. doi:10.1136/jamia.2010.004366

34 Dumitrescu L, Ritchie MD, Denny JC, et al. Genome-wide study of resistant hypertension identified from electronic health records. PLoS One 2017;12:e171745. doi:10.1371/journal.pone.0171745

35 Kho AN, Hayes MG, Rasmussen-Torvik L, et al. Use of diverse electronic medical record systems to identify genetic risk for type 2 diabetes within a genome-wide association study. J Am Med Informatics Assoc 2012;19:212-8. doi:10.1136/amiajnl-2011000439

36 Hindorff LA, Sethupathy P, Junkins HA, et al. Potential etiologic and functional implications of genome-wide association loci for human diseases and traits. Proc Natl Acad Sci U S A 2009;106:9362-7. doi:10.1073/pnas.0903103106

37 Kho AN, Pacheco JA, Peissig PL, et al. Electronic Medical Records for Genetic Research: Results of the eMERGE Consortium. Sci Transl Med 2011;3:79re1-79re1. doi:10.1126/scitranslmed.3001807

38 Denny JC, Ritchie MD, Basford MA, et al. PheWAS: demonstrating the feasibility of a phenome-wide scan to discover gene-disease associations. Bioinformatics 2010;26:1205-10. doi:10.1093/bioinformatics/btq126

39 How Common Is Breast Cancer? https://www.cancer.org/cancer/breast-cancer/about/how-commonis-breast-cancer.html (accessed 22 Apr 2018).

40 Paez JG, Jänne PA, Lee JC, et al. EGFR mutations in lung cancer: correlation with clinical response to gefitinib therapy. Science 2004;304:1497-500. doi:10.1126/science.1099314

41 Lynch TJ, Bell DW, Sordella R, et al. Activating Mutations in the Epidermal Growth Factor Receptor Underlying Responsiveness of Non-Small-Cell Lung Cancer to Gefitinib. N Engl J Med 2004;350:2129-39. doi:10.1056/NEJMoa040938

42 Jørgensen KJ, Gøtzsche PC, Kalager M, et al. Breast Cancer Screening in Denmark. Ann Intern Med 2017;166:313. doi:10.7326/M16-0270

43 Bollig-Fischer A. How the future of clinical cancer diagnostics can contribute to overcoming race-associated cancer disparities. Expert Rev Mol Diagn 2016;16:1233-5. doi:10.1080/14737159.2016.1254552

44 Bushel PR, Wolfinger RD, Gibson G. Simultaneous clustering of gene expression data with clinical chemistry and pathological evaluations reveals phenotypic prototypes. BMC Syst Biol 2007;1:15. doi:10.1186/1752-0509-1-15

45 Diaz D, Nguyen T, Draghici S. A Systems Biology Approach for Unsupervised Clustering of High-Dimensional Data. In: Machine Learning, Optimization, and Big Data. Springer, Cham 2016. 193 203. doi:10.1007/978-3-319-51469-7_16

46 Paoli S, Jurman G, Albanese D, et al. Integrating gene expression profiling and clinical data. Int J Approx Reason 2008;47:58-69. doi:10.1016/j.ijar.2007.03.012

47 Muzny DM, Bainbridge MN, Chang K, et al. Comprehensive molecular characterization of human colon and rectal cancer. Nature 2012;487:330-7. doi:10.1038/nature11252

48 Chin L, Andersen JN, Futreal PA. Cancer genomics: from discovery science to personalized medicine. Nat Med 2011;17:297-303. doi:10.1038/nm.2323

49 Boland MR, Hripcsak G, Shen Y, et al. Defining a comprehensive verotype using electronic health records for personalized medicine. J Am Med Inform Assoc 2013;20:e232-8. doi:10.1136/amiajnl2013-001932

50 Cancer Genome Atlas Research Network, Weinstein JN, Collisson EA, et al. The cancer genome atlas pan-cancer analysis project. Nat Genet 2013;45:1113.

51 Cancer Genome Atlas Research Network T, Koboldt DC, Fulton RS, et al. Comprehensive molecular portraits of human breast tumours. Nature Published Online First: 2012. doi:10.1038/nature11412

52 Cancer T, Atlas G, Koboldt DC, et al. Comprehensive molecular 
bioRxiv preprint doi: https://doi.org/10.1101/307751; this version posted April 25, 2018. The copyright holder for this preprint (which was not certified by peer review) is the author/funder. All rights reserved. No reuse allowed without permission.

Diaz et al., 24 Apr 2018 - preprint copy - BioRxiv

portraits of human breast tumours. Nature Published Online First: 2012. doi:10.1038/nature 11412

53 Kolda TG, Bader BW. Tensor Decompositions and Applications. SIAM Rev 2009;51:455-500.

54 Mørup M. Applications of tensor (multiway array) factorizations and decompositions in data mining. Wiley Interdiscip Rev Data Min Knowl Discov 2011;1:24-40. doi:10.1002/widm.1

55 Yener B, Acar E, Agius P, et al. Multiway modeling and analysis in stem cell systems biology. BMC Syst Biol 2008;2:63. doi:10.1186/1752-0509-2-63

56 Omberg L, Meyerson JR, Kobayashi K, et al. Global effects of DNA replication and DNA replication origin activity on eukaryotic gene expression. Mol Syst Biol 2009;5:312. doi:10.1038/msb.2009.70

57 Tucker LR. Some mathematical notes on three-mode factor analysis. Psychometrika 1966;31:279-311. doi:10.1007/BF02289464

58 Carroll JD, Chang J-J. Analysis of individual differences in multidimensional scaling via an n-way generalization of 'EckartYoung' decomposition. Psychometrika 1970;35:283-319. doi:10.1007/BF02310791

59 Harshman RA. Foundations of the PARAFAC procedure: Models and conditions for an 'explanatory' multi-modal factor analysis. UCLA Work Pap Phonetics 1970;:184.http://citeseerx.ist.psu.edu/viewdoc/download;jsessionid=B7D8 5530F5B04944CFFE01CB3F72983F?doi=10.1.1.144.5652\&rep=r ep1\&type=pdf (accessed 15 Feb 2018).

60 Saria S, Goldenberg A. Subtyping: What It is and Its Role in Precision Medicine. IEEE Intell Syst 2015;30:70-5. doi:10.1109/MIS.2015.60

61 Krämer A, Green J, Pollard J, et al. Causal analysis approaches in Ingenuity Pathway Analysis. Bioinformatics 2014;30:523-30. doi:10.1093/bioinformatics/btt703

62 Liu H, Patel MR, Prescher JA, et al. Cancer stem cells from human breast tumors are involved in spontaneous metastases in orthotopic mouse models. Proc Natl Acad Sci U S A 2010;107:18115-20.

63 Fillmore CM, Kuperwasser C. Human breast cancer cell lines contain stem-like cells that self-renew, give rise to phenotypically diverse progeny and survive chemotherapy. Breast Cancer Res 2008;10:R25. doi:10.1186/bcr1982

64 Dave B, Mittal V, Tan NM, et al. Epithelial-mesenchymal transition, cancer stem cells and treatment resistance. Breast Cancer Res 2012;14:202. doi:10.1186/bcr2938

65 Bao B, Mitrea C, Wijesinghe $\mathrm{P}$, et al. Treating triple negative breast cancer cells with erlotinib plus a select antioxidant overcomes drug resistance by targeting cancer cell heterogeneity. Sci Rep 2017;7:srep44125. doi:10.1038/srep44125

66 Acar E, Dunlavy DM, Kolda TG. A scalable optimization approach for fitting canonical tensor decompositions. J Chemom 2011;25:6786. doi:10.1002/cem. 1335

67 Chi EC, Kolda TG. On Tensors, Sparsity, and Nonnegative Factorizations. SIAM J Matrix Anal Appl Philadelphia 2012;33:1272-99.

68 Håstad J. Tensor rank is NP-complete. J Algorithms 1990;11:64454. doi:10.1016/0196-6774(90)90014-6

69 Bro R, Kiers HAL. A new efficient method for determining the number of components in PARAFAC models. J Chemom 2003;17:274-86. doi:10.1002/cem.801

70 Bro R, Kjeldahl K, Smilde AK, et al. Cross-validation of component models: A critical look at current methods. Anal Bioanal Chem 2008;390:1241-51. doi:10.1007/s00216-007-1790-1

71 Mørup M, Hansen LK. Automatic relevance determination for multi-way models. J Chemom 2009;23:352-63. doi:10.1002/cem.1223 